\title{
Diabetes mellitus tipo 2 y calidad de vida relacionada con la salud: resultados del Estudio Hortega
}

\author{
F. J. MENA MARTÍN, J. C. MARTÍN ESCUDERO, F. SIMAL BLANCO, J. BELLIDO \\ CASADO, J. L. CARRETERO ARES
}

Servicio de Medicina Interna. Hospital Universitario del Río Hortega. Valladolid

TYPE 2 DIABETES MELLITUS AND HEALTH-RELATED QUALITY

OF LIFE: RESULTS FROM THE HORTEGA STUDY

\section{RESUMEN}

Objetivo: Evaluar qué aspectos de la calidad de vida relacionada con la salud (CVRS) se ven afectados por la diabetes mellitus tipo 2 (DM), y la influencia del control glucémico y de los factores de riesgo cardiovascular (FRCV) asociados.

Método: Llevamos a cabo un estudio transversal descriptivo en el área sanitaria de nuestro hospital. Tras un muestreo polietápico analizamos una muestra final de 495 personas, representativa de la población general. La CVRS se valoró con el Short-Form 36 (SF-36).

Resultados: Tras ajustar por variables sociodemográficas (edad, sexo, nivel de estudios, estado civil, número de persones residiendo juntas, situación laboral, clase social y medio rural o urbano), comorbilidad y FRCV (tabaquismo, tensión arterial sistólica, LDL-colesterol e índice de masa corporal), los pacientes con DM presentaban menores puntuaciones en cuatro escalas del SF-36: función física, dolor corporal, salud general y vitalidad. Entre los diabéticos tensión arterial sistólica, índice de masa corporal y hemoglobina glicosilada se correlacionaban negativamente con algunas escalas, pero no así los niveles lipídicos.

Conclusiones: En nuestra población los pacientes con DM presentan una peor CVRS específicamente en las dimensiones físicas, sin que se vean afectadas ni su función social ni su salud mental. Hipertensión, obesidad y mal control glucémico se asocian en estos enfermos a un peor estado subjetivo de salud.

PALABRAS CLAVE: Calidad de vida. Diabetes mellitus tipo 2. Hipertensión. Obesidad. Hemoglobina glicosilada. Factores de riesgo.
ABSTRACT

Objective: To evaluate which health-related quality of life (HRQOL) aspects are affected by type 2 diabetes mellitus (DM) and influence of glycemic control and associated cardiovascular risk factors (CVRF).

Method: A descriptive cross-sectional study was carried out in the health coverage area of our hospital. Following a multiphase sampling a final sample of 495 people, representative of the general population, was analyzed. HRQOL was evaluated with the Short-Form 36 (SF-36).

Results: After adjustment for sociodemographic variables (age, sex, education level, marital status, number of persons residing together, labor situation, social class and rural or urban ambience), comorbidity and CVRF (smoking, systolic blood pressure, LDL-cholesterol level and body mass index), the patients with DM presented lower scores on four SF-36 scales: physical function, bodily pain, general health and vitality. Among people with diabetes systolic blood pressure, body mass index and glycosylated hemoglobin, but not lipid levels, were negatively correlated to some SF-36 scores.

Conclusions: In our population patients with DM present a poorer HRQOL, specifically in the physical dimensions, but neither their social function nor their mental health was affected. Hypertension, obesity and poor glycemic control are associated with worst subjective state of health.

KEY WORDS: Quality of Life. Diabetes mellitus type 2. Hypertension. Obesity. Hemoglobin A glycosylated. Risk factors.

Mena Martín FJ, Martín Escudero JC, Simal Blanco F, Bellido Casado J, Carretero Ares JL. Diabetes mellitus tipo 2 y calidad de vida relacionada con la salud: resultados del Estudio Hortega. An Med Interna (Madrid) 2006; 23: 357-360.

\section{INTRODUCCIÓN}

La mejoría de la calidad de vida relacionada con la salud (CVRS) representa el objetivo último de todas las intervenciones sanitarias en los pacientes con diabetes mellitus tipo 2 (DM). Tanto los síntomas de la propia enfermedad como los de sus complicaciones, y por supuesto las enfermedades asociadas, deterioran de forma significativa su capacidad funcio- nal y su sensación de bienestar (1); además el impacto emocional y social de la DM es muy importante y puede causar alteraciones psicológicas graves. Durante la década de los 90 el análisis de la CVRS se ha introducido gradualmente en los estudios de intervención terapéutica, de modo que actualmente todos los que se realizan contienen uno o varios cuestionarios de CVRS. Sin embargo se han llevado a cabo muy pocos estudios de CVRS con muestras no seleccionadas de pacien- 
tes, representativas de la población general diabética. Por otra parte es muy escasa la información disponible sobre qué aspectos de la CVRS se ven más deteriorados en estos pacientes, y los pocos trabajos disponibles muestran resultados muy dispares (1-3).

El objetivo del presente estudio es analizar, mediante el Medical Outcomes Study 36-item Short-Form Health Survey (SF-36), la CVRS de una muestra representativa de la población general (4) y, desde esta perspectiva plantear dos cuestiones: a) ¿qué dimensiones de la CVRS están deterioradas en los diabéticos?; en aquéllos con depresión o ansiedad es lógico esperar un deterioro en los aspectos psicológicos de la CVRS, pero ¿qué ocurre en el resto?, ¿hasta qué punto se ven afectados los aspectos físicos?; y b) ¿qué situaciones se asocian a un mayor deterioro de la CVRS?; se ha comprobado que el factor que condiciona en mayor medida dicho deterioro es la existencia de complicaciones (5); por otra parte la mayor parte de los estudios muestran que un mejor control glucémico se asocia con mejor CVRS (5) y reducción de los costes sanitarios (6); sin embargo se tienen pocos datos sobre el impacto de los factores de riesgo cardiovascular (FRCV) asociados sobre el estado subjetivo de salud de los pacientes con DM.

\section{MATERIAL Y MÉTODO}

\section{DISEÑO DEL ESTUDIO}

El diseño y la metodología detallados del estudio han sido ya publicados (4). En resumen, el trabajo se llevó a cabo en el área sanitaria del Hospital Universitario del Río Hortega (Valladolid), área que comprende una población de 214.445 personas. Seleccionamos una muestra de 1.500 sujetos a través de un muestreo aleatorio poliestratificado por todas las variables recogidas en una fase previa del estudio (edad, sexo, índice de masa corporal -IMC-, nivel de estudios y FRCV conocidos), con el objetivo de garantizar la máxima representatividad de la muestra y alcanzar la mayor precisión posible en las estimaciones referidas a los estratos más pequeños.

El estudio constó de un cuestionario enviado por correo, que incluía el SF-36, y una entrevista; también se extrajeron muestras de sangre, realizándose determinaciones de colesterol total, HDL-colesterol y triglicéridos mediante un autoanalizador Hitachi 917 (Boehringer); los niveles de LDL-colesterol se calcularon con la fórmula de Friedewald. En la entrevista se recogieron los siguientes datos: a) variables sociodemográficas, incluyendo la clase social basada en la ocupación del individuo según la British Registrar General classification $(7,8)$; b) otras enfermedades crónicas; y c) peso, altura y tensión arterial. Todos los participantes dieron su consentimiento para participar en el estudio. En los pacientes con DM se llevó a cabo también una determinación de hemoglobina glicosilada $\left(\mathrm{HbA}_{1 \mathrm{c}}\right)$ en una muestra de sangre capilar mediante el DCA $2000 \mathrm{HbA}_{l c}$ analyzer (Bayer Diagnostics, Tarrytown, NY).

Participaron un total de 495 individuos, 36 de los cuales eran diabéticos conocidos. Para descartar la existencia de algún tipo de sesgo se analizaron las historias clínicas de los 1.500 sujetos de la muestra, detectándose una mayor participación de los diabéticos en esta fase del estudio, con una menor prevalencia de DM entre los no participantes (4,55 vs. $7,41 \% ; p=0,016)$. No había diferencias entre participantes y no participantes respecto a ninguna de las otras variables analizadas, de la fase previa del estudio, o de la revisión de las historias clínicas (antecedentes de cardiopatía isquémica, arteriopatía peripérica o enfermedad cerebrovascular, glucemia basal, niveles de colesterol, tiempo de evolución de la DM y número de ingresos hospitalarios en los últimos cinco años).

\section{MEDIDAS}

El SF-36 contiene 36 ítems que valoran ocho aspectos de la CVRS: función física, limitaciones en el desempeño del rol por problemas físicos (rol físico), dolor corporal, percepción general del estado de salud, vitalidad, función social, limitaciones en el desempeño del rol por problemas emocionales (rol emocional) y salud mental. Se pueden obtener dos índices globales del cuestionario: el Physical Component Score (PCS) y el Mental Component Score (MCS) $(9,10)$.

\section{ANÁLISIS ESTADÍSTICO}

Dado que la muestra era estratificada los resultados se ponderaron según el peso de cada estrato, oscilando éste entre 0,4108 y 1,9161. Para examinar la correlación entre las escalas del SF-36 y tensión arterial sistólica (TAS), IMC y $\mathrm{HbA}_{1}$ se calcularon los coeficientes de correlación de Pearson (r) cuando las dos variables seguían una distribución normal y los de Spearman $\left(\mathrm{r}_{\mathrm{s}}\right)$ cuando alguna no seguía dicha distribución.

Se llevó a cabo un análisis descriptivo para determinar las diferencias entre diabéticos y no diabéticos respecto a las siguientes variables: factores sociodemográficos (edad, sexo, nivel de estudios, estado civil, número de personas residiendo juntas, situación laboral, clase social y medio rural o urbano), comorbilidad expresada como número de enfermedades no cardiovasculares asociadas, y FRCV: tabaquismo, TAS, LDLcolesterol e IMC. Todos estos factores y covariables fueron entonces incluídos en un modelo lineal general, y mediante el método post hoc de Bonferroni se testó la influencia independiente de la DM sobre la puntuación en las escalas del SF-36 (variables dependientes).

\section{RESULTADOS}

La tabla I muestra las características de los sujetos con y sin DM. Entre los primeros el 6,98\% estaban en tratamiento solamente con dieta, el $12,88 \%$ en tratamiento insulínico y el $80,14 \%$ restante sólo con hipoglucemiantes orales y dieta; el tiempo medio de evolución de la enfermedad era de 9,25 \pm 8,67 años, con un valor medio de $\mathrm{HbA}_{1 \mathrm{c}}$ de 7,8 $\pm 1,7 \%$.

En el modelo multivariable los pacientes con DM presentaban puntuaciones significativamente más bajas en cuatro de las ocho escalas del SF-36: función física, dolor corporal, salud general y vitalidad (Tabla II).

Entre los diabéticos el 6,39\% no tenían ningún otro FRCV asociado, es decir eran no fumadores, presentaban un buen control de la tensión arterial $(<130 / 80 \mathrm{mmHg})$, estaban en situación de bajo riesgo según sus niveles de triglicéridos, HDL y LDL-colesterol (11) y su IMC era $<30 \mathrm{~kg} / \mathrm{m}^{2}$; el 23,55\% tenían un FRCV asociado, el 68,01\% dos, y el 2,05\% tres FRCV. El número de FRCV asociados se correlacionaba 
TABLA I

CARACTERÍSTICAS DE LOS SUJETOS CON $(\mathrm{N}=36)$ Y SIN $(\mathrm{N}=$ 459) DIABETES MELLITUS TIPO 2

\begin{tabular}{|c|c|c|}
\hline & Sujetos con DM & Sujetos sin DM \\
\hline $\begin{array}{l}\text { Sexo (\% varones) } \\
\text { Edad (años) } \\
\quad \text { Rango } \\
\end{array}$ & $\begin{array}{c}58,3 \\
66,0 \pm 6,4 \\
56-80 \\
\end{array}$ & $\begin{array}{c}46,4 \\
44,0 \pm 16,2^{a} \\
15-82 \\
\end{array}$ \\
\hline $\begin{array}{l}\text { Estado civil (\%) } \\
\text { Casado } \\
\text { Soltero } \\
\text { Viudo } \\
\end{array}$ & $\begin{array}{c}86,1 \\
2,8 \\
11,1 \\
\end{array}$ & $\begin{array}{l}67,7 \\
28,6 \\
3,6^{b} \\
\end{array}$ \\
\hline $\begin{array}{l}\text { Nivel de estudios (\%) } \\
\text { Estudio universitarios } \\
\text { Educación básica/estudios de grado m } \\
\text { Educación básica incompleta } \\
\end{array}$ & $\begin{array}{r}13,9 \\
\text { medio } 8,3 \\
77,8\end{array}$ & $\begin{array}{l}29,2 \\
44,6 \\
26,2^{c} \\
\end{array}$ \\
\hline $\begin{array}{l}\text { Situación laboral (\%) } \\
\text { Jubilado } \\
\text { Ama de casa } \\
\text { Trabajador activo } \\
\text { Estudiante/parado }\end{array}$ & $\begin{array}{c}72,2 \\
16,7 \\
11,1 \\
0\end{array}$ & $\begin{array}{l}17,5 \\
20,3 \\
49,4 \\
12,9^{c}\end{array}$ \\
\hline Lugar de residencia (\% rural) & 22,2 & 25,8 \\
\hline $\begin{array}{l}\text { Clase social }(\%)^{e} \\
\text { I, II y III } \\
\text { IVa, IVb y V } \\
\text { VI }\end{array}$ & $\begin{array}{l}25,9 \\
36,2 \\
37,9 \\
\end{array}$ & $\begin{array}{l}41,9 \\
22,0 \\
36,1 \\
\end{array}$ \\
\hline Número de personas viviendo juntas & $2,4 \pm 1,6$ & $2,8 \pm 1,4$ \\
\hline $\begin{array}{l}\text { Hipertensión arterial conocida (\%) } \\
\text { Tensión arterial } \geq 140 / 90 \mathrm{mmHg}(\%)\end{array}$ & $\begin{array}{c}47,46 \\
57,6 \\
\end{array}$ & $\begin{array}{l}12,37^{c} \\
31,4^{b}\end{array}$ \\
\hline $\begin{array}{l}\text { Hipercolesterolemia conocida (\%) } \\
\text { LDL-colesterol (mg/dl) } \\
\text { HDL-colesterol (mg/dl) } \\
\text { Triglicéridos (mg/dl) }\end{array}$ & $\begin{array}{c}44,83 \\
120 \pm 33 \\
47 \pm 10 \\
117 \pm 61\end{array}$ & $\begin{array}{c}19,39^{c} \\
133 \pm 35^{d} \\
56 \pm 13^{\mathrm{a}} \\
92 \pm 45^{d}\end{array}$ \\
\hline Hábito tabáquico activo (\%) & 17,2 & 27,1 \\
\hline
\end{tabular}

Obesidad (\% con índice de masa corporal $\geq a$

\begin{tabular}{ccc}
$30 \mathrm{~kg} / \mathrm{m}^{2}$ ) & 30,56 & $11,49^{\mathrm{b}}$ \\
Índice de masa corporal & $28,40 \pm 3,85$ & $25,60 \pm 4,05^{\mathrm{a}}$ \\
\hline
\end{tabular}

DM: diabetes mellitus tipo 2 ; ${ }^{a} p<0,0001$, t de Student; ${ }^{b} p<0,01$, Chicuadrado; ${ }^{c} p<0,0001$, Chi-cuadrado; ${ }^{d} p<0,01$, t de Student; ${ }^{e}$ profesionales liberales, directivos y técnicos superiores; II técnicos medios, otros directivos, artistas y deportistas; III administrativos y cuadros y mandos intermedios; IVa trabajadores manuales cualificados; IVb trabajadores manuales semi-cualificados; $\mathrm{V}$ trabajadores no cualificados; VI ocupaciones no clasificables.

negativamente con la puntuación en las escalas de función social $\left(r_{\mathrm{s}}=-0,391 ; \mathrm{p}=0,015\right)$ y salud mental $\left(\mathrm{r}_{\mathrm{s}}=-0,359 ; \mathrm{p}=\right.$ 0,027). La relación entre las escalas del cuestionario y los FRCV era evidente en el caso de la hipertensión arterial (HTA) y la obesidad: la TAS se correlacionaba negativamente con la puntuación en vitalidad $(\mathrm{r}=-0,395 ; \mathrm{p}=0,022)$, función social $\left(r_{s}=-0,493 ; p=0,005\right)$, salud mental $(r=-0,459 ; p=$ $0,007)$ y MCS $(r=-0,403 ; p=0,021)$; por su parte el IMC se correlacionaba negativamente con la puntuación en rol físico $\left(r_{s}=-0,374 ; p=0,023\right)$, función social $\left(r_{s}=-0,496 ; p=0,004\right)$ y PCS $(\mathrm{r}=-0,339 ; \mathrm{p}=0,045)$; no había correlación entre las escalas del cuestionario y los niveles lipídicos.
El porcentaje de $\mathrm{HbA}_{1 \mathrm{c}}$ se correlacionaba negativamente con la puntuación en las escalas de función social $\left(r_{s}=-0,494 ; p\right.$ $=0,003)$, rol emocional $\left(r_{\mathrm{s}}=-0,350 ; \mathrm{p}=0,042\right)$ y salud mental $\left(r_{\mathrm{s}}=-0,460 ; \mathrm{p}=0,006\right)$, así como con el MCS $(\mathrm{r}=-0,450 ; \mathrm{p}=$ $0,008)$. Estas correlaciones seguían siendo significativas al analizar el subgrupo de diabéticos que referían no conocer qué valora la determinación de $\mathrm{HbA}_{1 \mathrm{c}}$ : función social $\left(\mathrm{r}_{\mathrm{s}}=-0,565 ; \mathrm{p}\right.$ $=0,001)$, rol emocional $\left(r_{s}=-0,517 ; p=0,004\right)$, salud mental $\left(r_{s}\right.$ $=-0,466 ; p=0,008)$ y MCS $(r=-0,443 ; p=0,014)$.

\section{DISCUSIÓN}

El presente estudio recoge información de una muestra de pequeño tamaño pero representativa de la población de nuestro medio. Se han llevado a cabo muy pocos estudios poblacionales sobre la CVRS de la población diabética (2,12-14), la mayoría se basan en muestras selecciondas de pacientes. La muestra que hemos analizado presenta un porcentaje relativamente bajo de diabéticos en tratamiento insulínico, porcentaje que en otros estudios llega a ser de hasta el 50-60\% al tratarse de muestras seleccionadas; esto aumenta la validez de nuestros resultados, teniendo en cuenta que la terapia insulínica se asocia a una peor CVRS en pacientes con DM (15).

Hemos utilizado un cuestionario genérico como el SF-36 $(9,10)$, de probada validez en muestras heterogéneas de individuos y en pacientes diabéticos $(12,15)$. Los estudios previos que han comparado la CVRS de personas con y sin DM mediante el SF-36 (2) o su versión previa ya en desuso, el SF$20(1,3)$, han incluído sólo variables sociodemográficas $(1,3)$ y comorbilidad (3) en el análisis multivariable; en nuestro trabajo hemos introducido también FRCV frecuentemente asociados a la DM tales como HTA, obesidad y dislipemia. De esta forma hemos comprobado que las personas diabéticas, además de un peor estado general de salud y menos vitalidad, muestran puntuaciones inferiores en dos escalas físicas, función física y dolor corporal, así como en el PCS; en otras palabras, en nuestra población las principales diferencias entre ambos grupos residen en las dimensiones físicas del concepto multidimensional de calidad de vida relacionada con la salud. Al igual que en el Medical Outcomes Study (1), la puntuación en la escala de salud mental en nuestro medio es similar en individuos con y sin DM; los autores que han detectado puntuaciones menores en dicha escala entre los diabéticos $(2,3)$ lo han atribuído a la alta prevalencia de depresión en estos pacientes (16). Por otra parte, mientras que en diversas poblaciones se ha descrito que la DM frecuentemente se asocia a una reducción de la vida social (17), con menores puntuaciones en la escala de función social $(1,3)$, nuestro estudio no ha revelado que la DM conlleve un deterioro de los aspectos sociales de la CVRS.

Una cuestión poco estudiada de la CVRS de la población diabética es la influencia de los FRCV asociados. Bradley et al mostraron que la obesidad se asocia a mayores nieles de depresión y ansiedad, así como a una menor satisfacción con el tratamiento (18). Por su parte Hanninen y cols. (3) detectaron que los pacientes con DM e IMC $\geq 32 \mathrm{~kg} / \mathrm{m}^{2}$ presentan una menor puntuación en la escala de función física del SF-20. En nuestra muestra el IMC se correlacionaba negativamente con rol físico y función social. Respecto a la influencia de HTA y dislipemia en la CVRS del diabético no hemos detectado en la revisión de la bibliografía datos al respecto; en nuestra población hemos 
TABLA II

\begin{tabular}{|c|c|c|c|c|c|}
\hline & Sujetos con DM (I) & Sujetos sin DM (J) & $\begin{array}{c}\text { Diferencias de } \\
\text { medias }(\mathrm{J}-\mathrm{I})\end{array}$ & $p$ & $\begin{array}{c}\text { Diferencias de } \\
\text { medias: IC 95\% }\end{array}$ \\
\hline Función física & $76,2 \pm 24,0$ & $87,8 \pm 50,0$ & 11,6 & 0,001 & $4,7-18,4$ \\
\hline Limitaciones de rol por problemas físicos & $73,3 \pm 51,3$ & $81,5 \pm 106,7$ & 8,2 & 0,266 & $-6,3-22,8$ \\
\hline Dolor corporal & $56,7 \pm 37,4$ & $68,6 \pm 77,7$ & 11,9 & 0,028 & $1,3-22,5$ \\
\hline Función social & $90,7 \pm 31,1$ & $85,9 \pm 64,6$ & $-4,8$ & 0,285 & $-13,6-4,0$ \\
\hline Limitaciones de rol por problemas emocionales & $84,2 \pm 55,1$ & $74,7 \pm 114,6$ & $-9,5$ & 0,233 & $-25,2-6,2$ \\
\hline Salud mental & $72,2 \pm 29,8$ & $72,5 \pm 62,0$ & 0,4 & 0,932 & $-8,1-8,8$ \\
\hline Physical Component Score & $42,6 \pm 11,3$ & $49,3 \pm 23,5$ & 6,7 & $<0,0001$ & $3,5-10,0$ \\
\hline Mental Component Score & $49,2 \pm 16,7$ & $47,0 \pm 34,8$ & $-2,2$ & 0,361 & $-7,0-2,5$ \\
\hline
\end{tabular}

DM: diabetes mellitus tipo 2. IC: intervalo de confianza. ${ }^{\mathrm{T}}$ Tras ajustar por las siguiente variables incluidas en el modelo: factores sociodemográficos (edad, sexo, nivel de estudios, estado civil, número de personas viviendo juntas, situación laboral, clase social y medio rural o urbano), factores de riesgo cardiovascular (tabaquismo, tensión arterial sistólica, LDL-colesterol e índice de masa corporal) y comorbilidad expresada como número de enfermedades asociadas no cardiovasculares. Los datos muestran media \pm desviación estándar.

verificado una correlación negativa entre TAS y vitalidad, función social y salud mental, sin encontrar relación entre CVRS y niveles lipídicos.

Queremos resaltar el hecho de que cuanto mayor era el valor de $\mathrm{HbA}_{1 \mathrm{c}}$ más baja era la puntuación en varias escalas del SF-36, lo cual seguía siendo evidente tras excluir a los sujetos que conocían la utilidad de dicha determinación. Esto sugiere que la asociación entre mal control glucémico y peor CVRS es independiente de que el paciente conozca o no su grado de control metabólico (12), es decir que el peor estado subjetivo de salud de los enfermos con niveles más elevados de $\mathrm{HbA}_{1 \mathrm{c}}$ se relaciona más bien con los síntomas derivados de la hiperglucemia.

\section{AGRADECIMIENTOS}

Queremos agradecer los comentarios realizados sobre este trabajo por parte del Dr. Jordi Alonso (Institut Municipal d'Investigació Médica, Barcelona).

\section{Bibliografía}

1. Stewart AL, Greenfield S, Hays RD, Wells K, Rogers WH, Berry SD, et al. Functional status and well-being of patients with chronic conditions. Results from the Medical Outcomes Study. JAMA 1989; 262: 907-13.

2. Lyons RA, Lo SV, Littlepage BNC. Comparative health status of patients with 11 common illnesses in Wales. J Epidemiol Community Health 1994; 48: 388-390.

3. Hanninen J, Takala J, Keinanen-Kiukaanniemi S. Quality of life in NIDDM patients assessed with the SF-20 questionnaire. Diabetes Res Clin Pract 1998; 42: 17-27.

4. Mena-Martin FJ, Martin-Escudero JC, Simal-Blanco F, Carretero-Ares JL, Arzua-Mouronte D, Herreros-Fernandez V. Health-related quality of life of subjects with known and unknown hypertension: results from the population-based Hortega study. J Hypertens 2003; 21: 1283-9.

5. Rubin RR, Peyrot M. Quality of life and diabetes. Diabetes Metab Res Rev 1999; 15: 205-18.

6. Wagner EH, Sandhu N, Newton KM, McCulloch DK, Ramsey SD, Grothaus LC. Effect of improved glycemic control on health care costs and utilization. JAMA 2001; 285: 182-9.

7. Leete R, Fox J. Registrar General's social classes: origins and uses. Population Trends 1977; 8: 1-7.

8. Alonso J, Pérez P, Sáez M, Murillo C. Validez de la ocupación como un indicador de la clase social, según la clasificación del British Registrar General. Gac Sanit 1997; 11: 205-13.

9. Ware JE, Snow KK, Kosinski M, Gandek B. SF-36 Health Survey Manual and interpretation guide. Boston: The Health Institute, New England Medical Center; 1993.
10. Unitat de Recerca en Serveis Sanitaris. Manual de puntuación de la versión española del Cuestionario de Salud SF-36. Barcelona: Institut Municipal d’Investigació Mèdica; 1998.

11. IDF Clinical Guidelines Task Force. Global Guideline for Type 2 diabetes. Brussels: International Diabetes Federation; 2005

12. Anderson RM, Fitzgerald JT, Wisdom KK, Davis WK, Hiss RG. A comparison of global versus disease-specific quality-of-life measures in patients with NIDDM. Diabetes Care 1997; 20: 299-305.

13. Keinanen-Kiukaanniemi S, Ohinmaa A, Pajunpaa H, Koivukangas P. Health related quality of life in diabetic patients measured by the Nottingham Health Profile. Diabet Med 1996; 13: 382-8.

14. Gulliford MC, Mahabir D. Relationship of health-related quality of life to symptom severity in diabetes mellitus: a study in Trinidad and Tobago. J Clin Epidemiol 1999; 52: 773-80.

15. Jacobson AM, De Groot M, Samson JA. The evaluation of two measures of quality of life in patients with type I and type II Diabetes. Diabetes Care 1994; 17: 267-74

16. Gavard JA, Lustman PJ, Clouse RE. Prevalence of depression in adults with diabetes. An epidemiological evaluation. Diabetes Care 1993; 16 : 1167-78.

17. Mayou R, Bryant B, Turner R. Quality of life in non-insulin-dependent diabetes and a comparison with insulin-dependent diabetes. J Psychosom Res 1990; 34: 1-11.

18. Bradley C, Lewis KS. Measures of psychological well-being and treatment satisfaction developed from responses of people with tablet-treated diabetes. Diabet Med 1990; 7: 445-51. 